\title{
REGULARITY OF WEAK SOLUTIONS TO CRITICAL EXPONENT VARIATIONAL EQUATIONS
}

\author{
KAREn K. UhlenBeCK ${ }^{1}$ And JefF A. ViaclovskY ${ }^{2}$
}

\begin{abstract}
We present a general method for proving regularity of weak solutions to variational equations with critical exponent nonlinearities. We will focus primarily on the $C^{\infty}$ regularity of $L_{2}^{2}$ solutions to a nonlinear fourth order variational equation in 4 dimensions. This equation was considered by Chang, Gursky, and Yang in [CGY99], where regularity was obtained only for minimizers using techniques from Morrey [Mor48] and Schoen-Uhlenbeck [SU82]. The methods in this paper apply to a more general class of critical exponent variational equations in $n$ dimensions with leading term a power of the Laplacian.
\end{abstract}

\section{Introduction}

We let $(M, g)$ be a compact Riemannian manifold of dimension $n=4$. We consider the functional $F: L_{2}^{2}(M) \rightarrow \mathbf{R}$ from [CGY99] defined by

$$
F[w]=\int_{M}\left[\gamma(\Delta w)^{2}+\left(\Delta w+\beta|\nabla w|^{2}\right)^{2}+D(\nabla w, \nabla w)+E(w)\right] d v o l,
$$

where $\beta, \gamma \in \mathbf{R}, \gamma \neq-1, E: \mathbf{R} \rightarrow \mathbf{R}$, and $D$ is a symmetric 2-tensor (note that our $F$ is more general than the functional considered in [CGY99] in that we allow the coefficient of $(\Delta w)^{2}$ to be negative). Furthermore, we assume that for some constants $c_{1}, c_{2}$, and $c_{3}$

$$
|E(x)| \leq c_{1} e^{c_{2}|x|},|D(v, v)| \leq c_{3}|v|^{2} \text {, and }\left|E^{\prime}(x)\right| \leq c_{1} e^{c_{2}|x|} .
$$

We compute the first variation of the functional $F$ :

$$
\begin{aligned}
\left.\frac{d}{d t} F[w+t \phi]\right|_{t=0}= & \int_{M}\left[2 \gamma \Delta w \Delta \phi+2\left(\Delta w+\beta|\nabla w|^{2}\right)(\Delta \phi+2 \beta \nabla w \cdot \nabla \phi)\right] d v o l \\
& +\int_{M}\left[D(\nabla w, \nabla \phi)+E^{\prime}(w) \phi\right] d v o l .
\end{aligned}
$$

Integrating by parts, we find that the Euler-Lagrange equations take the following form:

$$
\begin{aligned}
2(1+\gamma) \Delta^{2} w+2 \beta \Delta|\nabla w|^{2}-4 \beta \operatorname{div}\left[\left(\Delta w+\beta|\nabla w|^{2}\right) \nabla w\right] & \\
& =\operatorname{div}[D(\nabla w, \cdot)]-E^{\prime}(w)
\end{aligned}
$$

The main result in this paper is the following:

Received August 28, 2000.

1 Research supported in part by Sid Richardson Regents' Chair Fund, University of Texas system.

${ }^{2}$ Research supported in part by an NSF Postdoctoral Fellowship. 
Theorem 1.1. If $w \in L_{2}^{2}(M)$ is a critical point of the functional $F$ in (1.1), and the conditions (1.2) are satisfied, then $w \in C^{\infty}(M)$.

We now give an outline of the proof of Theorem 1.1. We take advantage of the divergence form of the equation by using negative Sobolev spaces. The equation then maps $L_{2}^{2} \rightarrow L_{-2}^{2}$. A bootstrap argument shows that if we have a solution in $L_{2}^{p}$ for any $p>2$, then it is smooth (Lemma 2.1).

For the next part of the proof, we just look at the equation under a microscope, and use the scale invariant norm $\|\Delta w\|_{L^{2}}+\|\nabla w\|_{L^{4}}$. The point here is that by using a powerful enough microscope, we may assume that this norm is as small as we want, and we may just work in the unit ball, $B_{1} \subset \mathbf{R}^{4}$. We then use a cutoff function, freeze coefficients, and view the equation as a linear equation. Since we are assuming the above norm is very small, we may then view the linear operator as a small perturbation of the biharmonic operator. Using a result on the solvability of the Dirichlet problem in $L_{2}^{p}$ (Lemma 3.1), we conclude our solution is in $L_{2}^{p}$ for some $p>2$. This part of the argument will be done in Section 3.

To end the introduction, we remark that this method applies to the regularity question for a more general class of variational equations with leading term a power of the Laplacian. We present some simple examples in Section 4.

\section{Bootstrap}

In this section we will prove the following:

Lemma 2.1. If $w \in L_{2}^{p}(M)$ is a weak solution of (1.3) for some $p>2$, then $w \in C^{\infty}(M)$.

Proof. We begin with some results on multiplication of functions in Sobolev spaces.

Proposition 2.1. If $1 \leq p<4$, then multiplication is a continuous multilinear map of

$$
\begin{array}{cc}
L_{1}^{p} \oplus L_{1}^{p} \rightarrow L_{1}^{q}, & q=\frac{4 p}{8-p} \\
L^{p} \oplus L_{1}^{p} \rightarrow L^{q}, & q=\frac{4 p}{8-p} \\
L_{1}^{p} \oplus L_{1}^{p} \oplus L_{1}^{p} \rightarrow L^{q}, & q=\frac{4 p}{3(4-p)} .
\end{array}
$$

If $4<p<\infty$, then

$$
\begin{gathered}
L_{1}^{p} \oplus L_{1}^{p} \rightarrow L_{1}^{p} \\
L^{p} \oplus L_{1}^{p} \rightarrow L^{p} \\
L_{1}^{p} \oplus L_{1}^{p} \oplus L_{1}^{p} \rightarrow L_{1}^{p} .
\end{gathered}
$$

Proof. The proof may be found in [Pal68].

By the Moser-Trudinger inequality ([Mos71], [Tru67]), if $w \in L_{2}^{2}$, then $e^{w} \in L^{p}$ for any $p>1$. Therefore by the assumptions (1.2), and Proposition 2.1, we find 
that if $2 \leq p<4$, equation (1.3) looks like (with a large abuse of notation)

$$
\Delta^{2} w+L_{-1}^{\frac{4 p}{8-p}}+L_{-1}^{\frac{4 p}{8-p}}+L_{-1}^{\frac{4 p}{3(4-p)}} \in L^{p} .
$$

If $2 \leq p<4$, then

$$
\frac{4 p}{3(4-p)} \geq \frac{4 p}{8-p}
$$

Therefore we conclude that

$$
\Delta^{2} w \in L_{-1}^{\frac{4 p}{8-p}} .
$$

From elliptic regularity and the Sobolev embedding theorem, we obtain

$$
w \in L_{3}^{\frac{4 p}{8-p}} \subset L_{2}^{\frac{2 p}{4-p}} .
$$

We see easily that we may iterate and get that $w \in L_{2}^{p^{\prime}}$ for some $p^{\prime}>4$.

Using the second part of Proposition 2.1, we find that equation (1.3) now looks like

$$
\Delta^{2} w+L_{-1}^{p^{\prime}}+L_{-1}^{p^{\prime}}+L^{p^{\prime}} \in L^{p^{\prime}}
$$

Therefore $\Delta^{2} w \in L_{-1}^{p^{\prime}}$, and by elliptic regularity we have $w \in L_{3}^{p^{\prime}}$. Since $p^{\prime}>4$, by the Sobolev imbedding theorem we conclude that $w \in C^{2, \alpha}$, for some $0<$ $\alpha<1$, and it follows easily that $w \in C^{\infty}$.

\section{Localization}

From the previous section, we must now show that if $w \in L_{2}^{2}(M)$ is a weak solution of (1.3), then $w \in L_{2}^{p}(M)$ for some $p>2$. First we will prove a local regularity result assuming that certain norms are small (Proposition 3.1), and then we will show this implies the full regularity result (Proposition 3.2). In what follows, we let $w \in L_{2}^{2}(M)$ be a weak solution of (1.3).

Proposition 3.1. Let $x_{0} \in M$, and let $B_{1}$ be the unit ball in a Riemannian normal coordinate system $\{x\}$ around $x_{0}$. Then there exists an $\epsilon>0$ such that

$$
\|\Delta w\|_{L^{2}\left(B_{1}\right)}+\|\nabla w\|_{L^{4}\left(B_{1}\right)}<\epsilon
$$

and

$$
\max _{i, j}\left\|g^{i j}-\delta^{i j}\right\|_{L^{\infty}\left(B_{1}\right)}<\epsilon,
$$

imply that $w \in L_{2}^{q}\left(B_{1 / 2}\right)$ for some $2<q<4$.

Proof. Recall that in Riemannian normal coordinates, $g_{i j}=\delta_{i j}+O\left(|x|^{2}\right)$ as $x \rightarrow x_{0}$. We note the following formula for the biharmonic operator:

$$
\Delta_{g}^{2} w=\frac{1}{\sqrt{g}} \partial_{i}\left(g^{i j} \sqrt{g} \partial_{j} \Delta_{g} w\right)=\frac{1}{\sqrt{g}} \partial_{i}\left(g^{i j} \sqrt{g} \partial_{j}\left(\frac{1}{\sqrt{g}} \partial_{k}\left(g^{k l} \sqrt{g} \partial_{l} w\right)\right)\right) .
$$


Expanding this out in the $\{x\}$ coordinates, we find the flat biharmonic operator (which we will denote by $\Delta_{0}^{2}$ ) plus some error terms, the worst of which contains fourth order derivatives of $w$, which we write in divergence form:

$$
\Delta_{g}^{2} w=\Delta_{0}^{2} w+\sum_{i, j, k, l} \partial_{i} \partial_{j}\left(a^{i j, k l} \partial_{k} \partial_{l} w\right)+\text { lower order terms, }
$$

where $a^{i j, k l}(x)=g^{i j}(x) g^{k l}(x)-g^{i j}(0) g^{k l}(0)=g^{i j}(x) g^{k l}(x)-\delta^{i j} \delta^{k l}$.

We choose a cutoff function $\phi \in C_{0}^{\infty}\left(B_{1}\right)$, with $\phi \equiv 1$ in $B_{1 / 2}$. We multiply equation (1.3) by $\phi$, let $h=\phi w$, and we obtain

$$
\begin{aligned}
2(1+\gamma)\left(\Delta_{0}^{2} h+L h\right)+2 \beta \Delta(\nabla w \cdot \nabla h)-4 \beta \operatorname{div}\left[\left(\Delta w+\beta|\nabla w|^{2}\right) \nabla h\right] \\
=f \in L_{-2}^{q}\left(B_{1}\right),
\end{aligned}
$$

for some $2<q<4$, where $L$ denotes the operator

$$
L h=\sum_{i, j, k, l} \partial_{i} \partial_{j}\left(a^{i j, k l} \partial_{k} \partial_{l} h\right) .
$$

We now freeze coefficients, view this as a linear equation in $h$, and estimate the terms in $L_{-2}^{p}$. For any $2<p_{0}<4$, and $p$ in the closed interval $2 \leq p \leq p_{0}$,

$$
\begin{aligned}
\|\Delta(\nabla w \cdot \nabla h)\|_{L_{-2}^{p}} & \leq C\|\nabla w \cdot \nabla h\|_{L^{p}} \leq C\|\nabla w\|_{L^{4}}\|\nabla h\|_{L^{\frac{4 p}{4-p}}} \\
& \leq C \epsilon\|h\|_{L_{1}^{\frac{4 p}{4-p}}} \leq C \epsilon\|h\|_{L_{2}^{p}},
\end{aligned}
$$

where $\epsilon$ denotes the maximum of the norms in (3.1) and (3.2), and the constants do not depend on $p$. Next we have (for the same $p$ ),

$$
\begin{aligned}
\|\operatorname{div}(\Delta w \nabla h)\|_{L_{-2}^{p}} & \leq C\|\Delta w \nabla h\|_{L_{-1}^{p}} \leq C\|\Delta w \nabla h\|_{L^{\frac{4 p}{p+4}}} \\
& \leq C\|\Delta w\|_{L^{2}}\|\nabla h\|_{L^{\frac{4 p}{4-p}}} \leq C \epsilon\|h\|_{L_{1}^{\frac{4 p}{4-p}}} \leq C \epsilon\|h\|_{L_{2}^{p}} .
\end{aligned}
$$

Similarly, we have

$$
\left\|\operatorname{div}\left(|\nabla w|^{2} \nabla h\right)\right\|_{L_{-2}^{p}} \leq C \epsilon^{2}\|h\|_{L_{2}^{p}} .
$$

Finally, we estimate the operator $L$. Since $a^{i j, k l}=O(\epsilon)$, we have

$$
\begin{aligned}
\|L h\|_{L_{-2}^{p}} & =\left\|\sum_{i, j, k, l} \partial_{i} \partial_{j}\left(a^{i j, k l} \partial_{k} \partial_{l} h\right)\right\|_{L_{-2}^{p}} \leq C \sum_{i, j, k, l}\left\|a^{i j, k l} \partial_{k} \partial_{l} h\right\|_{L^{p}} \\
& \leq C \epsilon \sum_{k, l}\left\|\partial_{k} \partial_{l} h\right\|_{L^{p}} \leq C \epsilon\|h\|_{L_{2}^{p}} .
\end{aligned}
$$

Since we are working in the unit ball, all of the Sobolev constants are fixed, so for $\epsilon$ sufficiently small, the equation (3.3) is a small perturbation of the equation

$$
\Delta_{0}^{2} h=f \in L_{-2}^{q}\left(B_{1}\right) .
$$

Lemma 3.1. The map

$$
\Delta_{0}^{2}: L_{2,0}^{p}\left(B_{1}\right) \rightarrow L_{-2}^{p}\left(B_{1}\right)
$$

is an isomorphism for $1<p<\infty$. 
Proof. This is just the Dirichlet problem in $L^{p}$ (see [Agm59]). The only subtlety is that we are using negative Sobolev spaces, but we note that the estimates in [Agm59] remain valid in this situation.

From the estimates (3.4), (3.5), (3.6), and (3.7) above, we conclude that for $\epsilon$ sufficiently small, the linear operator in (3.3) is also an isomorphism between $L_{-2,0}^{p}\left(B_{1}\right)$ and $L_{-2}^{p}\left(B_{1}\right)$ for $2 \leq p \leq p_{0}<4$. Choosing $p_{0}>q$, we may therefore solve equation (3.3) in $L_{2,0}^{q}\left(B_{1}\right)$. From uniqueness in $L_{2,0}^{2}\left(B_{1}\right)$, we conclude that $h \in L_{2,0}^{q}\left(B_{1}\right)$, and therefore $w \in L_{2}^{q}\left(B_{1 / 2}\right)$.

Proposition 3.2. If $w \in L_{2}^{2}(M)$ is a weak solution of (1.3), then $w \in L_{2}^{q}(M)$ for some $q>2$.

Proof. Let $x_{0} \in M$, and choose a Riemannian normal coordinate system $\{x\}$ around $x_{0}$, so that $g_{i j}=\delta_{i j}+O\left(|x|^{2}\right)$. For $0<r<1$, we will define the dilated coordinates by $\{\widetilde{x}=r x\}$, and let $T$ denote the change of coordinates $T: \widetilde{x} \rightarrow \widetilde{x} / r$. We consider the function $\widetilde{w}(\widetilde{x})=T^{*} w(\widetilde{x})=w(\widetilde{x} / r): B_{1} \rightarrow \mathbf{R}$, and define the metric $\widetilde{g}=r^{2} T^{*} g$. We multiply equation (1.3) by $r^{4}$, and we rewrite as

$$
\begin{aligned}
2(1+\gamma) \widetilde{\Delta}^{2} \widetilde{w}+2 \beta \widetilde{\Delta}|\widetilde{\nabla} \widetilde{w}|^{2}-4 \beta \widetilde{\operatorname{div}}[(\widetilde{\Delta} \widetilde{w} & \left.\left.+\beta|\widetilde{\nabla} \widetilde{w}|^{2}\right) \widetilde{\nabla} \widetilde{w}\right] \\
& =r^{2} \widetilde{\operatorname{div}}[D(\widetilde{\nabla} \widetilde{w}, \cdot)]-r^{4} E^{\prime}(\widetilde{w})
\end{aligned}
$$

where everything is taken with respect to the metric $\widetilde{g}$. We see that $\widetilde{w}$ satifies a similar equation in $B_{1}$ with respect to the $\widetilde{g}$ metric. The only difference are the terms on the right hand side. But since $r<1$ these terms are smaller than in the original equation, and Proposition 3.1 will of course remain valid for this dilated equation.

We note that the norm in (3.1) is scale invariant. That is, if we scale the metric by a constant, the norm does not change. Therefore for $r$ sufficiently small, we will have $\|\widetilde{\Delta} \widetilde{w}\|_{L^{2}\left(B_{1}\right)}+\|\widetilde{\nabla} \widetilde{w}\|_{L^{4}\left(B_{1}\right)}<\epsilon$.

Next we make the important observation that the $\{\widetilde{x}\}$ coordinates are a Riemannian normal coordinate system for the metric $\widetilde{g}$. Therefore, we have $\widetilde{g}^{i j}(\widetilde{x})=\delta^{i j}+O\left(|\widetilde{x}|^{2}\right)$, so by choosing $r$ sufficiently small, the quantity in (3.2) will also be smaller than $\epsilon$.

From Proposition 3.1, we conclude that $\widetilde{w} \in L_{2}^{q}\left(B_{1 / 2}\right)$, which implies that the original function $w \in L_{2}^{q}\left(B_{r}\right)$ for some $r<1 / 2$. We conclude that $w \in L_{2, l o c}^{q}(M)$, and therefore since $M$ is compact, $w \in L_{2}^{q}(M)$.

Remark. The estimates depend on the profile of $w \in L_{2}^{2}(M)$, and therefore are not uniform in the norm.

\section{Other equations}

The idea in this regularity argument works for other critical exponent problems (those in which the initial bootstrap argument fails) with leading term a 
power of the Laplacian. For example, the Yamabe equation in dimensions $n>2$ :

$$
L u=R u^{\frac{n+2}{n-2}},
$$

where $L$ is the conformal Laplacian, and $R$ is the scalar curvature. The corresponding problem here is to prove $C^{\infty}$ regularity for $L_{1}^{2}$ solutions (see [Tru68], where regularity is proved using a different argument). To adapt the above proof to this case, one exploits the dilational symmetry $u \mapsto r^{\frac{2-n}{2}} u(x / r)$, and the corresponding scale invariant norm $\|u\|_{L^{\frac{2 n}{n-2}}}$.

Another example is $Q$-curvature equation in dimensions $n>4$ :

$$
P u=Q u^{\frac{n+4}{n-4}},
$$

where $P$ is the generalized Paneitz operator, and $Q$ is the $Q$-curvature (see [CY97]). The corresponding problem is to prove $C^{\infty}$ regularity for $L_{2}^{2}$ solutions (in fact, the equation we considered previously in this paper is the four dimensional analogue of this equation with some additional nonlinearities). For this equation, one exploits the dilational symmetry $u \mapsto r^{\frac{4-n}{2}} u(x / r)$, and the corresponding scale invariant norm $\|u\|_{L^{\frac{2 n}{n-4}}}$.

\section{References}

[Agm59] S. Agmon, The $L_{p}$ approach to the Dirichlet problem. I. Regularity theorems, Ann. Scuola Norm. Sup. Pisa 13 (1959), 405-448.

[CGY99] S.-Y. A. Chang, M. J. Gursky, and P. C. Yang, Regularity of a fourth order nonlinear PDE with critical exponent, Amer. J. Math. 121 (1999), 215-257.

[CY97] S.-Y. A. Chang and P. C. Yang, Determinants and extremal metrics in conformal geometry, Geometry from the Pacific Rim (Singapore, 1994), de Gruyter, 1997, pp. 37-57.

[Mor48] C. B. Morrey, Jr., The problem of Plateau on a Riemannian manifold, Ann. of Math. 49 (1948), 807-851.

[Mos71] J. Moser, A sharp form of an inequality by N. Trudinger, Indiana Univ. Math. J. 20 (1970/71), 1077-1092.

[Pal68] R. S. Palais, Foundations of global non-linear analysis, W. A. Benjamin Inc., New York-Amsterdam, 1968.

[SU82] R. Schoen and K. Uhlenbeck, A regularity theory for harmonic maps, J. Differential Geom. 17 (1982), 307-335.

[Tru67] N. S. Trudinger, On imbeddings into Orlicz spaces and some applications, J. Math. Mech. 17 (1967), 473-483.

[Tru68] _ Remarks concerning the conformal deformation of Riemannian structures on compact manifolds, Ann. Scuola Norm. Sup. Pisa 22 (1968), 265-274.

Department of Mathematics, The University of Texas, Austin, TX 78712.

E-mail address: uhlen@math.utexas.edu

Department of Mathematics, Mit, Cambridge, MA 02139.

E-mail address: jeffv@math.mit.edu 